\title{
Tangence
}

\section{De la pratique des détours chez Sembène, Chamoiseau et Ben Jelloun}

\section{Christiane Ndiaye}

Numéro 49, décembre 1995

Les littératures francophones de l'Afrique et des Antilles

URI : https://id.erudit.org/iderudit/025878ar

DOI : https://doi.org/10.7202/025878ar

Aller au sommaire du numéro

Éditeur(s)

Tangence

ISSN

0226-9554 (imprimé)

1710-0305 (numérique)

Découvrir la revue

Citer cet article

Ndiaye, C. (1995). De la pratique des détours chez Sembène, Chamoiseau et Ben Jelloun. Tangence, (49), 63-77. https://doi.org/10.7202/025878ar d'utilisation que vous pouvez consulter en ligne.

https://apropos.erudit.org/fr/usagers/politique-dutilisation/ 


\section{De la pratique des détours chez Sembène, Chamoiseau et Ben Jelloun}

\section{Christiane Ndiaye}

L'espace qu'on attribue aujourd'hui à la francophonie est un espace extrêmement hétérogène. Même si l'on admet que la "vieille" francophonie, celle de l'Europe, se distingue de celle qui est issue de la période coloniale, on se trouve encore devant une pluralité culturelle irréductible. Entre le Québec, le Maghreb, l'Afrique et les Antilles francophones, où sont les lieux de convergence? Tout en reconnaissant le dénominateur commun qu'est la langue française, ceux qui appartiennent ou qui s'intéressent à cet espace tiennent actuellement un discours de plus en plus nuancé où les différences sont accentuées: malgré leur "antillanité", la culture haïtienne est autre que celle de la Martinique; la francophonie sénégalaise se distingue de celle du Cameroun qui n'est pas celle du Zaïre, etc. C'est ainsi qu'on a vu apparaître, depuis quelques années, de plus en plus d'ouvrages sur les littératures nationales. Dans ces conditions, comment enseigner "la littérature francophone"?

En effet, cette discipline existe et est peut-être mềme en expansion dans certaines institutions d'enseignement supérieur à travers le monde. En Amérique du Nord, on précise même, depuis quelque temps, qu'il s'agit de la francophonie "hors du Québec et de l'Europe». Le problème n'est pas pour autant réglé. L'enseignant à qui on confie la tâche de produire un cours d'introduction portant sur "la littérature francophone" se trouve à peu près aussi à l'aise que celui à qui on demanderait d'assurer un cours sur la littérature européenne. Nous sommes donc d'emblée appelés à relever le défi qui consiste à concilier l'un et le multiple.

Mais nous ne sommes ni les premiers ni les seuls. Les théoriciens de ces littératures nous ont précédés et ont proposé, au fil des années, plusieurs concepts permettant de faire un certain nombre de rapprochements sans gommer les différences. Les multiples variantes de la langue française ne font certes pas partie des racines des différentes cultures en question, mais elles peuvent être envisagées comme un de ces phénomènes rhizomatiques dont parle Glissant, lesquels relient les espaces divers de la 
64

francophonie ${ }^{1}$. En prenant pour exemple le trajet de Frantz Fanon, de Martinique en Algérie, Glissant conclut: "C'est bien là l'image du rhizome, qui porte à savoir que l'identité n'est plus toute dans la racine, mais aussi dans la Relation" ". Or, l'on sait que cette relation n'a pas été consentie, d'abord, mais imposée, dans le cas des anciennes colonies françaises. On s'accorde néanmoins pour dire que cette situation n'a pas eu que des conséquences néfastes et que, dans le domaine de la culture, notamment, une créativité particulière en est issue. Citant Houston A. Baker, Patrick Taylor, Wilson Harris et Derek Walcott, Simon Gikandi résume ainsi la démarche de l'écrivain colonisé: "colonial anxiety does not necessarily disable the colonized writer; rather, it enables a narrative liberation in the colonizer's language" 3 . Gikandi renvoie ensuite à Glissant qui a plus longuement expliqué cette appropriation subversive de la langue du colonisateur par le colonisé en s'appuyant, notamment, sur ce qu'il identifie comme des "pratiques du détour".

Confrontés dès leur arrivée au "nouveau monde" à des interdictions multiples, les esclaves ont dû faire preuve d'une inventivité exceptionnelle pour assurer la survie de leur communauté, corps et âmes. Conséquence:

L'apparition des contes, proverbes, dictons, chansons, tant dans le monde créolophone qu'ailleurs, est marquée du signe du discontinu. [...] On n'y trouve presque jamais la relation concrète des faits et gestes quotidiens, mais en revanche l'évocation symbolique des situations. Comme si ces textes s'efforçaient de déguiser sous le symbole, de dire en ne disant pas. C'est ce que j'ai appelé ailleurs une pratique du détour, et c'est en quoi le discontinu s'évertue; le même qui sera mis en acte par cet autre détour que fut le marronnage. ${ }^{4}$

1 Glissant se sert de cette distinction entre racine et rhizome (empruntée à Deleuze et Guattari) dans le développement de sa "poétique de la relation". Voir, entre autres, Édouard Glissant, "L'errance, l'exil ", dans Poétique de la relation, Paris, Gallimard, 1990 , p. 23-48.

2 Ibid., p. 31.

3 Simon Gikandi, Writing in Limbo. Modernism and Caribbean Literature, Ithaca et London, Cornell University Press, 1992, p. 12. Traduction: "l'angoisse coloniale n'entrave pas nécessairement l'écrivain colonisé; elle produit plutôt une libération narrative dans la langue du colonisateur ".

4 Édouard Glissant, op. cit., p. 82-83. Glissant aborde cette question également dans "Le retour et le détour" et "Poétique naturelle, poétique forcée " dans Le discours antillais, Paris, Seuil, 1981, p. 28-36, 236-253. 
Et la littérature écrite aurait poursuivi dans la même voie:

Ainsi les littératures de la Caraïbe, qu'elles soient de langue anglaise, espagnole ou française, introduisent-elles volontiers des épaisseurs et des cassures - comme autant de détours dans la matière dont elles traitent; mettant en pratique, à la manière du conte des Plantations, des procédés de redoublement, d'essoufflement, de parenthèse, d'immersion du psychologique dans le drame du devenir commun. La symbolique des situations y prévaut sur le raffinement des réalismes, c'est-à-dire qu'elle l'englobe, le dépasse et l'éclaire. ${ }^{5}$

Cette dernière précision est d'importance: englober, dépasser, éclairer n'est pas évacuer. Le "détour" implique toujours une certaine persistance du discours dominant. L'Autre a définitivement investi l'espace discursif, davantage même que l'espace physique. On ne peut l'en expulser, entièrement, mais on peut "l'englober" d'un autre langage qui produit alors une tout autre dynamique.

Il s'agit donc toujours de la coexistence de deux ou plusieurs modes d'expression, c'est-à-dire de plusieurs rapports au monde, plusieurs systèmes de valeurs différents. Le colonisé se réapproprie cet espace qui est le sien mais qui est occupé en même temps par l'Autre, ce qui l'amène inéluctablement à pratiquer cette "poétique de la relation" exposée par Glissant. Celle-ci est donc aussi une esthétique de l'ambivalence, au sens que prend ce terme chez Bakhtine (ambi-valence) dans ses études sur le carnavalesque ${ }^{6}$. Toutes les "pratiques du détour" ont pour effet de relativiser le discours dominant: les hiérarchies prescrites sont abolies; on se libère du carcan du dualisme exclusif. Il n'y a plus de dominant ni de dominé; il n'est plus nécessaire de choisir entre Être et Ne Pas Être. Les Différences coexistent et se multiplient.

Les "pratiques du détour " sont donc multiples et variées et on en retrouve à travers toutes les littératures nées du contexte colonial. Simon Gikandi choisit la figure emblématique de la danse "limbo" pour amorcer sa réflexion sur le modernisme des littératures antillaises. Cette danse aurait pris naissance dans les cales des bateaux négriers, où, déjà, les esclaves auraient trouvé le

5 Édouard Glissant, op. cit., p. 85.

6 Voir Mikhail Bakhtine, L'ceuvre de François Rabelais et la culture populaire au Moyen Âge et sous la Renaissance, trad. Andrée Robel, Paris, Gallimard, 1970, p. 19 et toute l'introduction, p. 9-67. 
66

moyen de manifester leur résistance et leur vitalité pour faire échec à la dépersonnalisation qui les guettait et s'approprier l'espace excessivement restreint alloué à cette "marchandise" par les trafiquants ${ }^{7}$. Dans ses études sur l'œuvre de l'écrivain nigérien Chinua Achebe, Gikandi donne un autre exemple, plus connu, de ce type de "détournement" de l'espace et des outils du maître réinvestis par les esclaves, soit l'emploi d'instruments européens pour créer la musique d'inspiration africaine qu'est le jazz ${ }^{8}$. De la même façon, les romanciers africains auraient fait du roman, genre éminemment européen, une "machine sémiotique" pour déplacer les idéologies hégémoniques et créer des systèmes de significations autres ${ }^{9}$. Achebe lui-même se serait fondé sur la métaphysique dualiste igbo pour effectuer ce "déplacement ":

Duality appeals to Achebe precisely because it produces a multiplicty of meanings and indeterminate zones of representation which generate narrative invention. In another sense, I believe, this duality allows the author, like his Igbo ancestors, to contest the central claims of Western metaphysics and its dependence on "Reason". ${ }^{10}$

Gikandi, comme Glissant, parlera alors des "détours " qu'inventent les écrivains colonisés pour circonvenir la culture imposée ${ }^{11}$.

Au Maghreb, le plus connu des "penseurs de l'ambivalence" est sans doute Abdelkebir Khatibi qui, dans plusieurs de ses écrits, trace à sa façon la voie d'une "pensée-autre", une pensée des différences intégrées:

Si donc l'Occident habite notre être intime, non point comme une extériorité absolue et dévastatrice, ni comme une maitrise

7 Simon Gokandi, op. cit., p. 14. Il s'agit d'une danse (pratiquée aux Antilles et en Amérique) où il faut passer sous une barre maintenue de plus en plus près du sol.

8 Simon Gikandi, Reading Cbinua Acbebe, Londres, Portsmouth et Nairobi, James Currey \& Heinemann, 1991, p. 22.

9 Dans le texte: "the novel allows the author to produce a semiotic machine that displaces hegemonic ideologies and creates alternative systems of meaning ". Ibid., p. 22.

10 Ibid., p. 20. Traduction: «La dualité plaît à Achebe précisément parce qu'elle crée une multiplicité de significations et de zones indétérminées de représentation qui génèrent l'inventivité narrative. Dans un autre sens, à mon avis, cette dualité permet à l'auteur, comme à ses ancêtres igbo, de contester les principaux présupposés de la métaphysique occidentale qui dépend de la "raison" ".

11 "These narratives are also forms of finding a detour around "the forms and

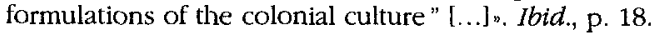


éternelle, mais bel et bien comme une différence, un conglomérat de différences à poser en tánt que tel dans toute pensée de lá différence et d'où qu'elle vienne; si donc l'Occident (ainsi nommé, ainsi situé) n'est pas la réaction à un désarroi incalculé, alors tout reste à penser : questions silencieuses qui souffrent en nous. ${ }^{12}$

Ainsi, Khatibi aussi prône le risque d'une pensée plurielle de l'altérité, une pensée "à plusieurs pôles de civilisation, à plusieurs langues, à plusieurs élaborations techniques et scientifiques" 13 . Une pensée de la marge, surtout, qui exclut toute domination:

Sur la scène planétaire, nous sommes plus ou moins marginaux, minoritaires et dominés. Sous-développés, disent-ils. C'est cela même notre chance, l'exigence d'une transgression à déclarer, à soutenir continuellement contre n'importe quelle autosuffisance. Bien plus, une pensée qui ne s'inspire pas de sa pauvreté est toujours élaborée pour dominer et humilier; une pensée qui ne soit pas minoritaire, marginale, fragmentaire et inacbevée, est toujours une pensée de l'ethnocide. ${ }^{14}$

Pensée des marges, pratiques du détour, conglomérat de différences: autant de "rhizomes" qui relient les différents espaces de la francophonie et qui permettent, malgré tout, de les réunir, à l'occasion, dans un même enseignement. On se limitera à trois exemples, en guise d'illustration: une nouvelle de l'écrivain sénégalais, Ousmane Sembène, "Le Voltaïque", Solibo Magnifique de Patrick Chamoiseau de la Martinique et quelques romans du Marocain Ben Jelloun, notamment L'enfant de sable et La nuit sacrée.

L'Afrique subsaharienne, si elle semble être moins portée vers les théorisations abstraites, a pourtant élaboré à sa façon cette pensée de la pratique des détours ${ }^{15}$. Il n'y a pas de meilleur exemple que cette nouvelle peu connue de Sembène qui se lit comme l'illustration parfaite du "marronnage créateur" dont parle Glissant ${ }^{16}$. Le récit met en scène un groupe de jeunes gens qui, au moment des indépendances africaines, s'interrogent sur l'origine des balafres que portent beaucoup d'Africains mais qui ne se

\footnotetext{
12 Abdelkebir Khatibi, Maghreb pluriel, Paris, Denoël, 1983, p. 12.

13 Ibid., p. 14.

14 Ibid., p. 17-18.

15 Voir, par exemple, les différentes déclarations faites par l'écrivain congolais, Sony Labou Tansi, en entrevue et dans ses écrits.

16 Édouard Glissant, op. cit.,p. 85.
} 
68

retrouvent pas chez les noirs des Antilles et d'Amérique. L'explication est fournie pas le Voltaique du titre qui raconte l'histoire suivante. À l'époque de la traite des esclaves (vers le milieu du $\mathrm{XvIII}^{\mathrm{e}}$ siècle, environ), une petite fille du nom de Iomé est enlevée de son village à l'intérieur du pays et amenée vers la côte où elle est enchaînée dans une cale de bateau négrier en attendant que le capitaine "fasse le plein". Pour la sauver, son père, Amoo, se joint à une bande de chasseurs d'esclaves qui entreprend de piller le bateau, pour s'emparer des marchandises et des esclaves, dans le but de revendre ceux-ci à d'autres trafiquants. Or, le chef de la bande, Momutu, n'a nullement l'intention de laisser repartir Amoo et sa fille qui représentent pour lui des marchandises de choix pour lesquelles il compte obtenir un bon prix. Toutefois, comme Amoo est aussi un guerrier intrépide, Momutu lui propose d'abord de se joindre à lui, pensant en faire un habile "chasseur" avant de le vendre, le moment venu. Et, de prime abord, l'argument dont il se sert pour obtenir la collaboration d'Amoo paraît assez convaincant :

- Tu n’as plus personne là-bas [au village], dit Momutu qui enchaîna avant qu'Amoo ait répondu: Moi aussi javais un village... [...] On mangeait de la viande... du poisson. Les champs étaient bien cultivés. Mais au fil cles ans, le village périclita. Ce n'était que lamentation sur lamentation. Depuis ma naissance je n'ai entendu que cris, n'ai vu que fuite éperdue dans la brousse, la forêt. Tu entres dans la forêt, tu meurs de maladie... Tu restes sur la savane... Tu es captif. Que puis-je faire? J'ai fait mon choix. J'aime mieux être avec les chasseurs quavec les chassés. ${ }^{17}$

Mais Amoo n'est pas dupe; sachant que Momutu ne cherche qu'à se servir de lui, il préfère prendre le risque de rejoindre son village.

Profitant d'une escarmouche avec d'autres acquerats, Amoo tue l'homme de Momutu chargé de le surveiller et s'enfuit avec Iomé pour retourner chez lui. Les villageois, pour échapper au danger qui les guette, décident alors de trouver un nouvel emplacement pour le village, plus loin dans la forêt. Cependant, avant que les préparatifs pour le départ ne soient achevés, le village est à nouveau la cible des aquerats. Tous s'enfuient dans la forêt, mais Amoo, qui tient à amener avec lui Iomé ainsi que sa belle-

17 Ousmane Sembène, Voltaique, Paris, Présence africaine, 1962, p. 205-206. 
mère, une vieille femme infirme, comprend rapidement que la situation est sans espoir. Alors lui vient une idée insolite. Il prend la petite fille et, avec son couteau, lui taillade la peau sur tout le corps. Les chasseurs d'esclaves, lorsqu'ils rattraperont les fugitifs, ne s'empareront que d'Amoo, puisque les trafiquants exigent une marchandise "impeccable . Iomé sera soignée par sa grand'mère; ses plaies se cicatriseront; elle portera des balafres, mais elle restera en liberté. Et bientôt la nouvelle se répand: la pratique est adoptée par des communautés entières qui échapperont ainsi à l'esclavage.

Pratique du détour exemplaire. Les villageois, ne pouvant expulser les prédateurs, ont néanmoins repris possession de leur espace, en l'occurrence leurs corps. C'est par une pensée-autre qu'ils se soustrairont au raisonnement élémentaire par lequel on cherche à les piéger et selon lequel il n'y a que deux modes d'être: on est maître ou esclave. Grâce à cet usage audacieux qu'Amoo fait de son couteau, ils ne seront ni chasseurs, ni chassés. Signes incontournables, les balafres les protégeront, comme les pratiques supposément magiques des communautés de marrons des Antilles ${ }^{18}$. Et quelle est cette magie? N'est-elle pas aussi celle de l'écriture-autre, cette écriture dans la langue de l'oppresseur qui est vécue d'abord comme une violence exercée contre soi-même mais qui permet malgré tout de se libérer de l'emprise dépersonnalisante de l'Autre? Une langue, comme un couteau, peut tuer, mais, manipulée autrement, elle peut aussi servir à déployer des énergies créatrices.

L'on voit qu'effectivement la "symbolique des situations prévaut sur le raffinement des réalismes", ici, comme le prévoyait Glissant. En introduisant dans cette fable des destinataires de l'Afrique des indépendances, Sembène ne met-il pas déjà en garde cette nouvelle génération contre les tentations d'une reproduction intégrale du système colonial avec une substitution simple qui mettra des Momutu à la place des "envahisseurs"? De ce point de vue, la nouvelle se lit comme une invitation à affirmer - par détour - sa Différence, à l'intérieur même de ce carcan oppressif où il n'y a de place que pour des "dominants " et des "dominés „... car cette conception du monde Autre n'est pas,

18 On appelle "Marrons" ceux qui s'enfuient des plantations pour échapper à l'esclavage. Ils vivent cachés dans les forêts des mornes, seuls ou en groupes. 
justement, une "extêriorité absolue", pour reprendre le mot de Khatibi ${ }^{19}$. Elle est bien implantée dans cet espace à soi, désormais composite, qu'on ne peut alors réinvestir que par les marges, par des pratiques qui "englobent" et "dépassent" les Chasseurs. Les règles du jeu sont perturbées; il n'y a plus ni gagnants, ni perdants.

Une dynamique analogue se développe dans le roman Solibo Magnifique de l'écrivain martiniquais Patrick Chamoiseau où deux visions du monde radicalement opposées coexistent, sans s'annuler. Au départ, le roman délimite deux espaces aussi hétérogènes que le sont le bateau négrier et le village en brousse chez Sembène. Le lecteur découvre d'abord la Savane, place publique de Fort-de-France. La Savane est l'espace du peuple, là où l'on se rencontre pour causer et se reposer un moment; c'est un espace ouvert où chacun est libre d'aller et venir à sa guise et où l'on rencontre à l'occasion Solibo, le conteur, accompagné du tam-tam de Sucette. C'est l'espace du rire, de la fête, de la fraternisation, de la liberté de mouvement et de parole, c'est-à-dire l'espace du minoritaire, du marginal, du fragmentaire et de l'inachevé qu'évoque Khatibi. L'histoire est d'ailleurs située au moment du carnaval; elle commence une nuit où Solibo, pour la première fois depuis longtemps, décide de se produire à la Savane devant un petit public un peu fatigué et quelque peu éméché, mais heureux des retrouvailles avec le Magnifique, ce maître de la parole à détours (à redoublements, essoufflements et parenthèses). Seulement Solibo meurt subitement, en pleine prestation, devant son auditoire, de ce qu'on dit être une "égorgette de la parole".

Interviennent alors les personnages qui appartiennent à l'autre espace, celui de la domination et de l'humiliation: l'hôtel de police. Évidemment, c'est un autre univers, un univers en tous points semblable à celui du chef esclavagiste, Momutu. Y règnent la loi et l'intérêt de l'Autre, la logique cartésienne (selon l'Inspecteur principal, Évariste Pilon), l'interdiction, la violence et la terreur. Autant la Savane est l'espace de la vie (comme l'était le village d'Amoo), autant l'Hôtel de Police est l'espace de l'oppression, de l'aliénation et de la mort.

19 L'on sait que Yambo Ouologuem, dans son roman Le devoir de violence, prétend que cela n'a jamais été le cas. 
Chacun de ces deux espaces a, par ailleurs, son langage propre et son souverain. Du côté de la police, c'est Évariste Pilon qui règne, mais c'est le brigadier-chef, Bouaffesse (frère jumeau de Momutu, ce "roitelet" de la traite ${ }^{20}$, qui se charge d'appliquer la loi de la manière forte. Les deux se prennent naturellement pour des surhommes, mais le texte ne tarde pas à les rabaisser, si bien qu'à la fin du roman ces deux figures d'autorité sont complètement démystifées. En fait, du point de vue du lecteur, ils sont ridicules dès le départ puisqu'ils décident de mener une enquête pour meurtre, tandis que le lecteur et le public de Solibo savent déjà qu'il s'agit d'une mort en quelque sorte surnaturelle - ce qui, dans le cas de Solibo, constitue une mort naturelle. Le comportement des policiers devient ainsi rapidement absurde; leur discours perd toute crédibilité.

Et pendant que les agents de la domination sont très vite rapetissés aux yeux du lecteur, Solibo le conteur, tout mort qu'il est, devient de plus en plus magnifique, occupant tous les interstices du récit. Chaque témoin, au lieu d'apporter les indices qu'exigent les policiers pour appuyer leur théorie, rend hommage, à sa façon, à cet homme du peuple qui était tout sauf ordinaire. Peu à peu, le texte crée un portrait "en mosaïque " ${ }^{21}$ dont chaque pièce contribue à rehausser l'image du conteur. Solibo, comme tout le monde, a traversé toutes les misères mais il s'est relevé, à l'aide de la parole ancienne, et il s'est ensuite efforcé de rendre l'existence de chacun un peu plus supportable - par sa parole, simplement, cette parole peu ordinaire qui peut envoûter les êtres humains mais aussi les bêtes et les êtres inhumains appartenant à l'espace policier ${ }^{22}$. Ainsi Solibo se transforme, au fil du texte, en une figure légendaire surhumaine: non seulement possède-t-il cet extraordinaire don de la parole, mais on lui prête aussi une sagesse plutôt occulte et on le considère comme un nègre marron des temps modernes qui refuse de s'intégrer à l'ordre établi. Tout cela le transforme en un être merveilleux qui peut apporter un peu de joie de vivre et de bonheur au sein de l'existence moribonde de tout un chacun. Il rebâtit ceux qu'il côtoie et les libère, "de l'intérieur". À la fin du roman, les deux hommes de la loi, sans disparaître, sont devenus tout à fait

\footnotetext{
20 Voir la description du personnage, Ousmane Sembène, op. cit., p. 206.

21 Cf. Patrick Chamoiseau, Solibo Magnifique, Paris, Gallimard, 1988, p. 26.

22 Voir ibid., p. 158-59.
} 
72

insignifiants, tandis que Solibo règne en Roi Magnifique sur le carnaval de la vie qui, grâce à lui, a repris un peu ses droits, ainsi que cela se produit après les interventions d'Amoo, dans la nouvelle de Sembène. La loi du plus fort est contournée, détournée, mise en échec, au point où Evariste Pilon lui-même sera amené, finalement, à reconnaître les valeurs qu'incarne Solibo.

Cette relativisation est marquée, d'autre part, par la présence des deux textes courts qui encadrent le récit proprement dit et qui sont en quelque sorte des échantillons exemplaires des langages qui caractérisent les deux univers représentés dans le roman. Le premier texte est le procès-verbal établi par Pilon concernant "le meurtre". Le texte de la fin est présenté comme une sorte d'approximation écrite des "Dits de Solibo". À la première lecture, le procès-verbal paraît clair et précis et les "Dits de Solibo" plutôt obscurs et incompréhensibles et on est tenté d'en faire abstraction. À la réflexion, on s'aperçoit cependant que ces deux séquences n'ont rien d'accessoire et qu'il faut les lire non pas séparément mais ensemble pour en saisir les significations qui, encore une fois, sont plutôt d'ordre symbolique. Or, lorsqu'on a pris connaissance de toute l'histoire de Solibo ainsi que de ses "dits " transcrits, et qu'on relit le procès-verbal policier, il apparaît que celui-ci est tout sauf ce qu'il prétend être: un résumé exact, objectif ("réaliste "), scientifique des faits. Dans le procès-verbal, Solibo apparaît comme un misérable vagabond qui est mort sous un arbre, au milieu d'un tas de détritus. Lorsqu'on compare cette version avec l'ensemble des témoignages, il est clair que la Vérité est ailleurs, s'il y en a une. Ce ne sont pas les paroles de Solibo qui paraissent alors insensées, mais plutôt ce langage officiel qui prétend établir les faits. Ainsi, le texte de Chamoiseau, comme celui de Sembène, met le lecteur en garde contre la représentation d'une situation proposée (imposée) par ceux qui occupent les instances du pouvoir. Il vaudrait mieux toujours s'écarter un peu pour ne pas s'y laisser prendre. Il n'y a pas que l'Histoire officielle; il y a aussi toutes ces petites histoires "fragmentaires " qui sortent de la bouche du peuple et qui réinventent la vie, constamment, au lieu de propager la violence et la mort.

Il est à noter, également, que l'ensemble du roman ne peut pas être lu comme une apologie sans réserve de toute forme d'oralité, c'est-à-dire un réquisitoire contre l'écrit en tant que tel, comme certaines remarques attribuées au personnage de Solibo pourraient le laisser croire. En effet, Solibo sermonne le narrateur 
en lui disant: "Cesse d'écrire kritia kritia, et comprends: se raidir, briser le rythme, c'est appeler sa mort.. Ti-Zibié, ton stylo te fera mourir couillon..." 23 . C'est pourtant Solibo qui meurt de sa propre parole - faute de public ${ }^{24}$. Le texte illustre ainsi, d'une autre manière, le danger de se cantonner dans des positions tranchées, quelles qu'elles soient. Solibo lui-même reconnaît d'ailleurs la nécessité de faire évoluer la tradition orale et de l'adapter à sa propre réalité vécue, en refusant, par exemple, de reproduire simplement des contes de compère Tigre et autres "africanités " qui n'existent pas aux Antilles ${ }^{25}$. Sa parole est une parole inventée de toutes pièces, donc, qui transgresse en fait les conventions de l'oral... tout comme l'écriture de Chamoiseau lui-même transgresse les conventions de l'écrit "réaliste" en intégrant plusieurs principes associés habituellement à l'oral (les procédés de cassure, de redoublement, etc. dont fait état Glissant).

Au niveau de l'écriture du texte lui-même, il s'agit donc de poser plusieurs modes de communication différents et de s'en servir également dans une démarche de synthèse, comme l'indiquent clairement les remarques de Glissant citées en exergue:

Je suis d'un pays où se fait le passage d'une littérature orale traditionnelle, contrainte, à une littérature écrite, non traditionnelle, tout aussi contrainte. [...] J'évoque une synthèse, synthèse de la syntaxe écrite et de la rythmique parlée, de l'"acquis" d'écriture et du "réflexe" oral, de la solitude d'écriture et de la participation au chanter commun - synthèse qui me semble intéressante à tenter. ${ }^{26}$

Dans le cas de Chamoiseau, une telle tentative de "co-production" a donné naissance à cette écriture d'une texture sans doute "baroque", mais riche et dynamique, que nous avons sous les yeux $^{27}$.

Des procédés de relativisation analogues, faisant appel au "détour", se retrouvent aussi dans les productions littéraires

23 Patrick Chamoiseau, op. cit., p. 76.

24 Cf. ibid., p. 224.

25 Voir ibid., p. 236.

26 Ibid., p. 11.

$27 C f$. l'étude de Delphine Perret, "Lire Chamoiseaun, où l'auteur examine le caractère composite de cette écriture et le lectorat pluriel qu'elle interpelle. Dans Maryse Condé et Madeleine Cottenet-Hage, Penser la créolité, Paris, Karthala, 1995, p. 153-172. 
74

maghrébines. Chez Ben Jelloun, autre représentant illustre de cette francophonie, "l'esthétique de l'ambivalence" est également mise en cuvre avec finesse. À sa façon, Ben Jelloun évoque la présence lancinante de l'Autre, dont on ne peut se défaire, mais qu'on peut déjouer, intégrer dans "un conglomérat de différences". De nouveau, le lecteur est convié à circuler dans un espace qui se dédouble pour s'ouvrir. Comme dans les deux autres textes, il se heurte à l'intransigeance des "souverains" qui règnent sur la politique, l'économie, la religion, etc. Chez eux, tout est figé, corrompu et dépourvu de vitalité. Dans tous les romans de Ben Jelloun, "l'univers des notables", comme il l'appelle, est un univers caractérisé par des formes géométriques, par la pierre et l'acier, par des murs et des certitudes immuables qui emprisonnent; c'est un univers où prévalent le conformisme, la mesquinerie et l'hypocrisie. Les personnages évoluent dans un milieu bourgeois, soi-disant "civilisé", mais qui impose en réalité des limites déshumanisantes à l'être humain. Cependant, cet ordre établi rigide occupe en fait assez peu de place dans les textes de Ben Jelloun; à travers toute son œuvre, Ben Jelloun développe surtout de multiples stratégies de perturbation destinées à minimiser l'impact des structures rigides associées à l'Autre dominateur.

Il convient de noter, par exemple, que les personnages principaux des romans de Ben Jelloun n'appartiennent jamais à "l'univers des notables "28. Ce sont des "demi-civilisés", des marginaux, des "infirmes sociaux": des mendiants, des fous, des orphelins, des prostituées, des vagabonds, des révoltés, des handicapés, des sorcières, etc. Ce ne sont jamais de bons citoyens bien pensants, "normalement" constitués. Ils sont tous informes ou difformes ou infirmes de quelque manière et ils vivent "dans les marges", en dehors des espaces qu'occupent les gens "civilisés", comme c'est le cas du "petit peuple" de la Savane, chez Chamoiseau. Ainsi que l'explique Zahra, dans L'enfant de sable:

Dans une société morale, bien structurée, non seulement chacun est à sa place, mais il n'y a absolument pas de place pour celui ou celle, surtout celle qui, par volonté ou par erreur, par esprit rebelle ou par inconscience, trahit l'ordre. ${ }^{29}$

28 Sauf peut-être celui de son dernier roman, L'bomme rompu, et même celui-ci s'y trouve plutôt malgré lui.

29 Tahar Ben Jelloun, L'enfant de sable, Paris, Seuil, 1985, p. 154. 
Toutefois, cette trahison, ce "marronnage", est évidemment valorisé chez Ben Jelloun. À travers la description et le comportement des personnages, il se développe un paradigme de l'inculte, de l'informe ou du difforme qui est en fait une expression de vitalité. Il vaut mieux être gros, laid, aveugle ou androgyne que trop beau et bien portant. Et l'on ne peut manquer de constater à quel point cette imagerie de la "résistance" rejoint celle des balafres chez Sembène.

Tous ces "Marrons" du quotidien rejettent ainsi la culture officielle et les lois sociales pour se rapprocher de la nature, qui exerce sur eux une influence salutaire désaliénante. Comme Solibo, dans le roman de Chamoiseau, ces êtres marginaux fuient les espaces clos urbains; ils ont rarement un domicile fixe. Ils se déplacent sans cesse, errent et voyagent à travers des espaces ouverts où la nature reprend ses droits ${ }^{30}$ : places publiques, terrains vagues, cimetières, déserts et autres paysages caractérisés tantôt par un lac, par des arbres, une source, le sable et la mer, etc. Tous ces déplacements accomplissent des rapprochements avec la nature et s'accompagnent d'une (re)découverte de soi, comme l'illustre bien l'épisode de la république des enfants dans La nuit sacrée 31 . Autrement dit, les personnages font de multiples détours pour échapper à "l'autosuffisance" (Khatibi), pour se dépasser et dépasser les cadres sociaux et/ou moraux trop bien structurés. Les formes géométriques de l'ordre établi sont donc complètement submergées et deviennent en quelque sorte inoffensives au sein de cet univers immoral et désordonné qui se crée autour des personnages de Ben Jelloun. Là encore, il apparaît que le processus est tout à fait analogue à celui du texte de Chamoiseau où l'enquête policière est "neutralisée" par une profusion d'histoires-autres.

Mais comme dans les deux autres textes étudiés, toute cette manifestation de vitalité ne semble pas avoir pour objectif d'éliminer complètement les figures et attributs qui caractérisent l'Ordre, ou, du moins, n'y parvient pas. Chez Ben Jelloun, c'est surtout la double nature des personnages qui en fait preuve: Moha est à la fois raisonnable et fou, vieillard et enfant; Harrouda et Yamna

30 Cela correspond exactement au cheminement de Solibo, tel que l'expliquent ceux (celles) qui racontent l'origine de son don de parole exceptionnel. Voir Patrick Chamoiseau, op. cit., p. 77-79.

31 Tahar Ben Jelloun, La muit sacrée, Paris, Seuil/France Loisirs, 1987, p. 40-50. 
sont tantôt des jeunes filles innocentes, tantôt de vieilles prostituées; le Consul peut être tyrannique mais avec sa sœur il redevient enfant et avec Zahra il est l'amant parfait, sensible. Zahra elle-même, après avoir retrouvé sa nature de femme, aime encore parfois jouer à l'homme ${ }^{32}$. Les marginaux et les tyrans, l'ordre et le désordre, l'ancien et le nouveau coexistent, au sein même d'un seul personnage, bien souvent. Il y a bien une prolifération de phénomènes fluctuants et mouvants, mais cela ne fait que minimiser ou dynamiser les éléments rigides; ils ne sont pas abolis. L'une des plus belles images de Ben Jelloun, qui traduit admirablement cette ambivalence, est celle du sable. Le sable est à la fois solide et fluide. Un grain de sable a des formes géométriques indestructibles, mais, emporté par le vent du désert, il crée des. dunes, des formes multiples et changeantes.

Par ailleurs, chez Ben Jelloun comme chez les deux autres écrivains, la mise en ouvre de ces diverses "pratiques du détour" s'accompagne d'une réflexion (plus ou moins explicite) sur l'écriture. Les textes de Ben Jelloun évoquent assez souvent des livres, des manuscrits, et d'autres textes écrits, mais on a tendance à s'en méfier. C'est que l'écriture aussi est composée de formes géométriques et lorsqu'elle relève du Coran et des textes de lois officiels, elle est associée aux interdictions et abus de pouvoir. Mais l'univers imaginaire de Ben Jelloun n'élimine pas pour autant l'écriture et les livres: tout comme Chamoiseau, il cherche plutôt à les "humaniser ", ainsi qu'en fait preuve la "bibliothèque humaine" dont il est question dans La nuit sacrée ${ }^{33}$. Ce que le lecteur peut en déduire, c'est qu'il n'est pas question de détruire les bibliothèques; on transforme plutôt cette institution en y mettant des corps humains, de jolies femmes qui récitent les ouvres apprises par cour. Séduisant détour. Ainsi s'explique aussi, d'une certaine façon, la valorisation de la voix et du conte dans l'œuvre de Ben Jelloun. Le conte (oral ou écrit) est étroitement rattaché à des sujets humains: quelqu'un parle àquelqu'un. Le texte se transmet à l'aide de la voix et du corps, la voix étant une sorte d'extension subtile du corps humain qui conserverait, mieux que

32 Cf. la dernière scène du roman, La nuit sacrée, p. 181. Rappelons que le roman, à la suite de L'enfant de sable, raconte l'histoire d'une jeune fille déguisée et élevée en garçon par son père qui ne peut se résigner à ne pas avoir d'héritier mâle. 
l'écrit, le dynamisme de la parole. Ainsi l'écriture de Ben Jelloun, comme celles de Chamoiseau et de Sembène, valorise cette rencontre des modes d'expression différents et s'en inspire, pour le plus grand bonheur du lecteur.

Il ne s'agit donc pas d'écrire comme l'Autre, ou bien de se taire, de ne pas écrire. Il s'agit d'écrire à sa manière, avec tous ces détours qui font la Différence. On s'empare de ce qui est donné (ou imposé) et l'on joue au plus rusé; on déjoue les mécanismes de la langue, des conventions de l'Autre.

Mais toute langue n'est-elle pas toujours "étrangère "34, même pour celui qui la croit "maternelle"? Toute langue n'est-elle pas déjà une "bilangue"?

[...] le bilinguisme interne à toute langue (celui du communicable à l'incommunicable, de la "prose" à la poésie) opère une séparation, un acte de scission, de différence et de transmutation selon un mouvement qui ne cesse de se doubler et de se dédoubler. Et le texte littéraire (tout texte) serait la scénographie de cette énonciation bificle. La langue française n'est pas la langue française: elle est plus ou moins toutes les langues internes et externes qui la font et la défont. ${ }^{35}$

Seulement, en "métropole", on peut (veut) parfois l'oublier, tandis qu'ailleurs, où le besoin de faire "reculer l'indicible " 36 est une urgence vécue quotidiennement, tous apprennent vite à reconnầtre les "failles" de la langue et tiennent à s'en servir, comme on a pu le constater à travers cette lecture de quelques textes de la francophonie. Ainsi, ne serait-ce que parce que "l'énonciation bifide" propre à tout texte littéraire est particulièrement marquée dans les ceuvres de la francophonie, il sera toujours instructif et enrichissant d'enseigner "la littérature francophone". Combien de lecteurs "unilingues" ont conscience de cette multiplicité qui foisonne dans leur propre langue et qui ne demande qu'à "marronnern?

34 Roland Barthes, cité par Tahar Bekri: ade toute manière, toute langue est étrangère". Dans "Le dur métier qu'est l'écriture", Itinéraires et contacts des cultures, vol. 10, "Littératures maghrébines ", t. 1, Paris, L'Harmattan, 1989, p. 75 .

35 Abdelkebir Khatibi, op. cit., p. 188.

36 Ibid., p. 190. 